Rubens Antonio da Silva 1

Vera Lúcia Cortiço Corrêa Rodrigues 2

Maria Esther de Carvalho 1

Clóvis Pauliquévis Jr. 3

\section{Programa de Controle da Doença de Chagas no Estado de São Paulo: persistência de alta infestação por triatomíneos em localidades na década de 1990}

\author{
Chagas Disease Control Program \\ in the State of São Paulo: persistence of high \\ triatomine infestation rates in some localities \\ during the 1990s
}

1 Superintendência de Controle de Endemias. Rua Paula Souza 166, São Paulo, SP 01027-000, Brasil. rubens@sucen.sp.gov.br esther@sucen.sp.gov.br

2 Laboratório de Parasitoses por Flagelados,

Superintendência de Controle de Endemias. Rua Afonso Pessini 86, C. P. 192, Mogi Guaçu, SP 13840-970, Brasil. verana@dglnet.com.br 3 Serviço Regional de Araçatuba, Superintendência de Controle de Endemias. Rua Minas Gerais 135, Araçatuba, SP 16015-060, Brasil. sro9@sucen.sp.gov.br

\begin{abstract}
The purpose of our investigation was to analyze the persistence, in the State of São Paulo, of domiciliary units highly infested with Chagas disease triatomine vectors. Comprehensive epidemiologic information collected from 1990 to 1999 in the State of São Paulo was retrieved from database files. Consistently high infestation rates were found in 43 localities distributed among 21 municipalities in the Araçatuba region. Captures produced predominantly peridomiciliary Triatoma sordida specimens, mostly adult females and fifth-instar nymphs, 94.6\% of which containing ingesta negative for human blood. The houses, mostly inhabited (87.4\%), were located near the residual woodland savanna in $64.3 \%$ of the localities. Poultry coops were found in association with $97.6 \%$ of all domiciliary units. Interestingly, in all the houses with persistent infestation, the inhabitants paid no evident attention to hygiene Lack of timely infestation control associated with the biological characteristics of the reduviid species involved, along with the conditions prevailing around the dwellings, favor the repeated invasion and consequent maintenance of colonization by triatomines.
\end{abstract}

Key words Chagas Disease; Vector Control; Triatomine

Resumo O objetivo, aqui, é relatar a persistência, em localidades do Estado de São Paulo, de alta infestação de triatomíneos vetores da doença de Chagas. Analisaram-se bancos de dados com informações trabalhadas para todo o Estado no período de 1990 a 1999. Em 43 localidades, distribuídas em 21 municípios concentrados na região de Araçatuba, observaram-se, sempre, altos índices de infestação. A espécie predominante foi Triatoma sordida com presença marcante no peridomicílio. Os exemplares capturados, em sua maioria adultos fêmeas e ninfas de quinto estádio, foram negativos para ingesta de sangue humano em 94,6\% dos casos. As casas eram habitadas $(87,4 \%)$ e encontravam-se próximas a resíduos de mata de cerrado em $64,3 \%$ das localidades. Observou-se presença de galinheiros em 97,6\% das Unidades Domiciliares. Ressalta-se que, nas casas de 100\% das localidades com infestação persistente, os moradores não cuidavam da limpeza. O atraso na realização do controle, associado às características biológicas da espécie e às condições dos peridomicílios são fatores que favorecem a invasão constante deste ambiente por triatomíneos e, conseqüentemente, a manutenção da infestação.

Palavras-chave Doença de Chagas; Controle de Vetores; Triatomíneos 


\section{Introdução}

O controle da transmissão vetorial da doença de Chagas no Estado de São Paulo, Brasil, teve início em 1950 com as atividades dirigidas ao combate do Triatoma infestans, considerado o principal vetor para a transmissão da doença. Com resultados bastante satisfatórios em relação ao ambiente domiciliar, esta espécie foi controlada e os triatomíneos mais freqüentemente coletados no peridomicílio passaram a assumir maior importância (Souza et al., 1984; Wanderley, 1993). As atividades de vigilância entomológica e controle da doença de Chagas desenvolvidas pela Superintendência de Controle de Endemias (SUCEN) passaram a ser desencadeadas sobre as espécies secundárias que colonizam preferencialmente o peridomicílio, sendo esta uma das características das espécies presentes atualmente no Estado. Com a ausência de capturas de T. infestans, incorporaram-se readequações racionalizadoras de atividades. Em 1990, implantou-se uma nova estratégia de controle (SES-SP, 1989). Classificaram-se as localidades em prioridades de acordo com o índice de infestação encontrado: Prioridade $1(\mathrm{P} 1)$ - localidades com infestação intradomiciliar (II) $\geq 5$ e ou infestação peridomiciliar (IP) $\geq 10$ (infestação alta); Prioridade 2 (P2) - localidades com II $<5$ e IP $<10$ (infestação média) e Prioridade 3 (P3) - localidades com II e IP = 0 (infestação nula). Nas localidades classificadas em P1 e P2, realizam-se atividades de pesquisa integral (rotina) de todas as casas e anexos (Unidade Domiciliar - UD). No primeiro caso, trabalham-se todas as casas e, no segundo, apenas uma amostra. Independentemente da classificação destas localidades atendem-se as notificações de triatomíneos encaminhadas pela população. No local de encontro de exemplares de triatomíneos, realiza-se o controle químico. Onde o controle químico é necessário, programam-se novas visitas. Os resultados das atividades de rotina e atendimento às notificações são revertidos em índices que irão determinar, bienalmente, a prioridade de cada localidade do Estado.

A vigilância triatomínica está direcionada a todas as espécies que porventura invadam ou colonizem as casas. No Estado de São Paulo, Triatoma sordida tem sido a espécie capturada, com maior freqüência nas regiões de Ribeirão Preto, São José do Rio Preto e Araçatuba. Estas áreas coincidem com as da distribuição de Rhodnius neglectus. A segunda espécie mais freqüentemente encontrada é o Panstrongylus megistus, compreendendo áreas florestadas das regiões de Sorocaba, Campinas, municípios li- mítrofes da região de Ribeirão Preto com o Estado de Minas Gerais e região litorânea.

Em localidades do Estado têm sido observados índices de alta infestação por triatomíneos ao longo dos anos, com manutenção em sua classificação epidemiológica. O objetivo deste estudo é relatar as características das UDs nas localidades onde se observou alta infestação por triatomíneos em toda a década de 1990.

\section{Material e métodos}

Analisaram-se dados secundários em bancos que contêm as informações trabalhadas para todo o Estado de São Paulo no Programa de Controle da Doenças de Chagas (PCDCh) desenvolvido pela SUCEN para o período de 1990 a 1999. A partir de 1990, as ações de vigilância preconizam atividades de pesquisa integral de captura de triatomíneos (rotina) e atendimento de notificação por parte da população em localidades do Estado. A periodicidade de pesquisa rotineira no PCDCh é bienal. Os resultados alcançados nestas pesquisas são revertidos em índices, que determinam a periodicidade de trabalho. Consideram-se os índices obtidos em atividade de rotina: $\mathrm{II}=$ casas positivas/casas pesquisadas $\mathrm{X} 100 \mathrm{ou} \mathrm{IP}=$ peridomicílios positivos/peridomicílios pesquisados X 100 e os de atividade de atendimento às notificações: II = atendimentos positivos no intradomicílio / casas existentes na localidade X 100 ou IP = atendimentos positivos no peridomicílio/casas existentes na localidade X 100. Estes determinarão a classificação das localidades e o trabalho a desenvolver para o biênio seguinte.

As localidades, em relação às suas prioridades, foram comparadas bienalmente. Selecionaram-se, para o estudo, as que apresentaram infestação alta (II $\geq 5$ e/ou IP $\geq 10$ ) em todos os biênios.

As informações sobre as características das UDs como proximidade à mata, presença de anexo e tipo de construção da moradia foram extraídas de boletins da SUCEN Estudo de Localidade.

\section{Resultados}

Das 14.900 localidades existentes no Estado de São Paulo, cerca de 5,0 \% estiveram classificadas em P1 no período de 1990 a 1999 (Figura 1). Neste período, observou-se uma alta infestação por triatomíneos em 43 localidades distribuídas em 21 municípios, 18 dos quais concen- 
Figura 1

Programa de Controle da Doença de Chagas, São Paulo, Brasil, década de 1990.

Municípios com localidades classificadas em Prioridade 1.

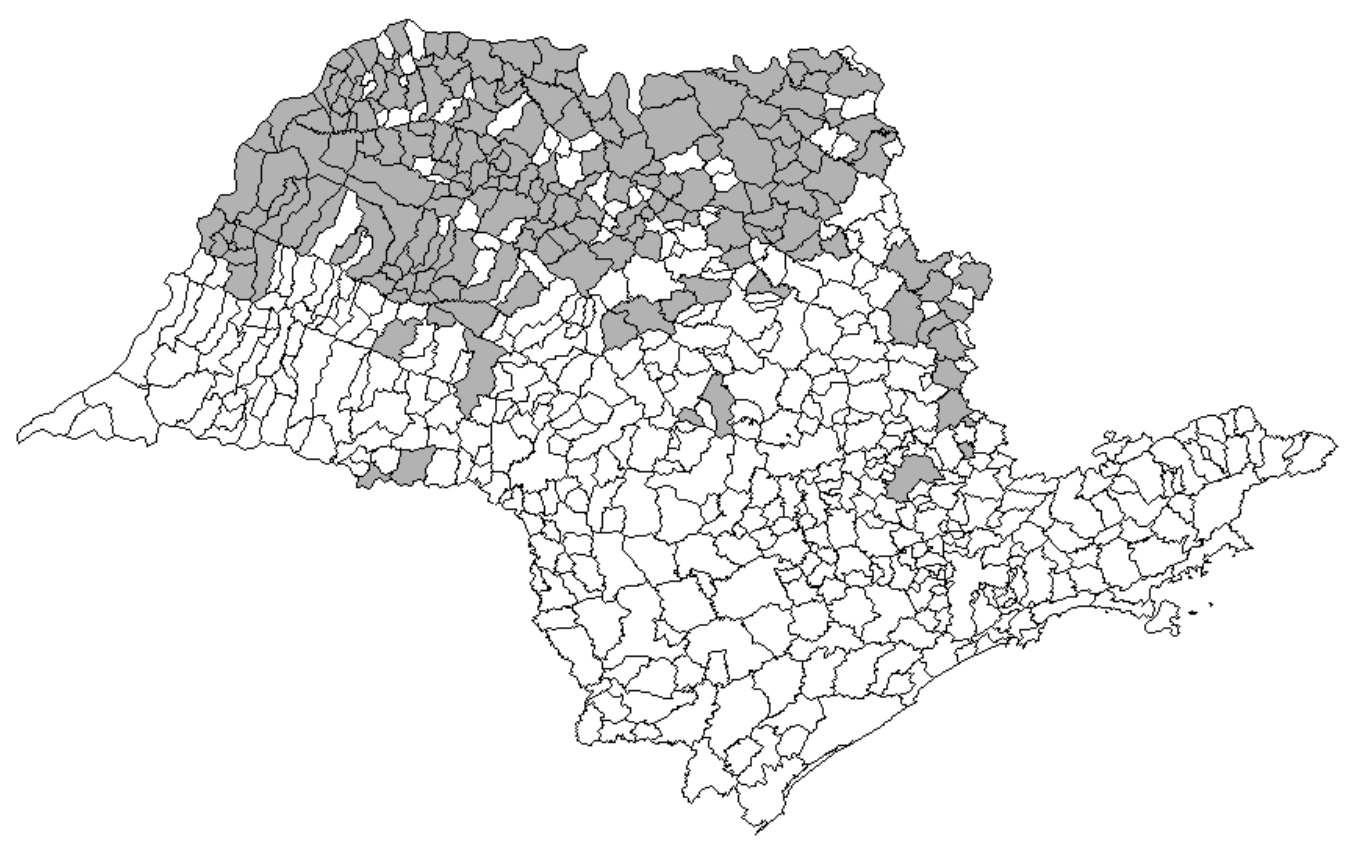

Municípios com localidades com alta infestação.

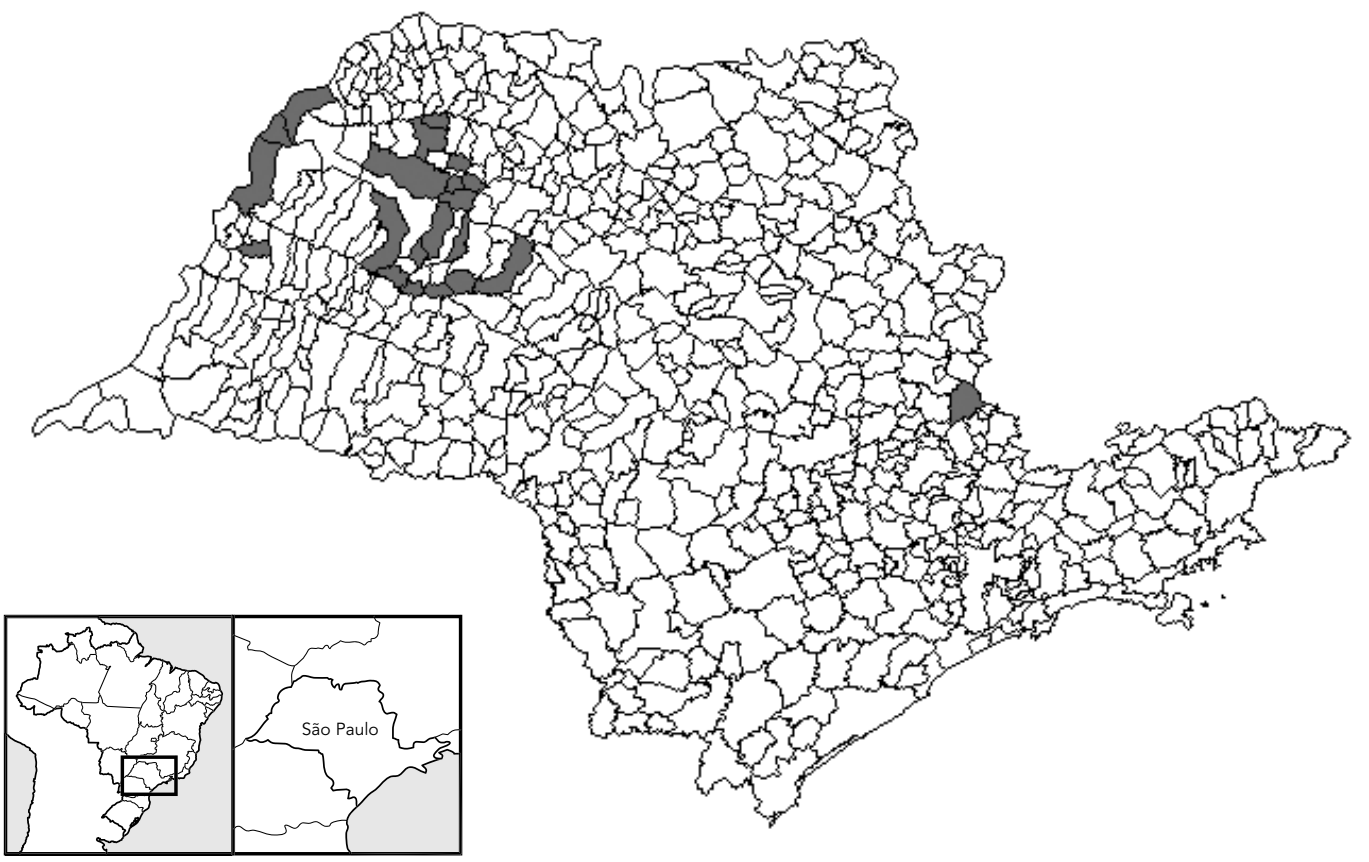

Fonte: DOT/SUCEN. 
trados na região noroeste do Estado - região de Araçatuba (Figura 1). A vegetação predominante nesta região é o cerrado com pequenos resíduos de matas ciliares. Boa parte do terreno é utilizada para pecuária, principal atividade agrícola da área, onde as pastagens se alternam com áreas de cerrado.

Na região de Araçatuba, com concentração de localidades com alta infestação, encontramos as espécies $R$. neglectus e T. sordida, com predomínio da segunda, capturadas, em sua maioria, no peridomicílio $(56,0 \%)$. As capturas ocorreram ao longo do ano, com maior concentração nos meses de julho, agosto e setembro.

Coletaram-se, nas UDs das localidades com alta infestação, no período em questão, 30.861 triatomíneos, sendo a maioria dos exemplares ninfas de quinto estádio e adultos fêmeas $(28,1 \%$ e $25,7 \%$, respectivamente). Observaram-se resultados negativos para ingesta de sangue humano em $97,7 \%$ dos exemplares capturados nestas localidades.

De acordo com a Tabela 1, podemos verificar que $8,9 \%$ das localidades apresentaram alto índice de infestação na década de 1990. Este percentual cai para 0,3 , quando calculado sobre o universo das localidades do Estado. Em 48,6\% das UDs houve captura de triatomíneos em apenas uma ocasião no transcorrer da década de 1990. A atividade de rotina respondeu pelo maior número de casas positivas se comparada à de notificação pelo morador, porém com menor eficiência, pois a cada três notificações recebidas pela população, uma foi positiva, ao passo que, na atividade de rotina, a cada 10 casas pesquisadas, uma foi positiva.

Tabela 1

Caracterização dos municípios nas localidades onde se observou alta infestação por triatomíneos na década de 1990.

Programa de Controle da Doença de Chagas, Estado de São Paulo, Brasil.

\begin{tabular}{|c|c|c|c|c|c|c|c|c|c|c|c|c|c|}
\hline \multirow[t]{3}{*}{ Município } & \multirow{3}{*}{$\begin{array}{l}\text { Espécie } \\
\text { predomi- } \\
\text { nante }\end{array}$} & \multirow{2}{*}{\multicolumn{3}{|c|}{ Localidades }} & \multicolumn{9}{|c|}{ Localidades com alta infestação } \\
\hline & & & & & \multicolumn{3}{|c|}{ Número de casas } & \multicolumn{3}{|c|}{ Notificações } & \multicolumn{3}{|c|}{ Atividade de rotina } \\
\hline & & Exist. & $\begin{array}{c}\text { Alta } \\
\text { infestação }\end{array}$ & $\%$ & Exist. & Positivas & $\%$ & Recebidas & positivas & $\%$ & $\begin{array}{c}\text { Casas } \\
\text { pesqui- } \\
\text { sadas }\end{array}$ & $\begin{array}{c}\text { Casas } \\
\text { positivas }\end{array}$ & $\%$ \\
\hline Alto Alegre & T. sordida & 32 & 2 & 6,2 & 53 & 36 & 67,9 & 28 & 7 & 25,0 & 236 & 27 & 11,4 \\
\hline Bilac & T. sordida & 12 & 3 & 25,0 & 114 & 47 & 41,2 & 24 & 15 & 62,5 & 509 & 43 & 8,4 \\
\hline Birigüi & T. sordida & 40 & 3 & 7,5 & 137 & 85 & 62,0 & 79 & 38 & 48,1 & 668 & 96 & 14,3 \\
\hline Buritama & T. sordida & 19 & 2 & 10,5 & 57 & 34 & 59,6 & 11 & 5 & 45,4 & 259 & 39 & 15,0 \\
\hline Castilho & T. sordida & 33 & 3 & 9,1 & 145 & 81 & 55,9 & 44 & 13 & 29,5 & 626 & 85 & 13,5 \\
\hline Gastão Vidigal & T. sordida & 15 & 1 & 6,7 & 39 & 29 & 74,3 & 5 & 2 & 40,0 & 187 & 36 & 19,2 \\
\hline $\begin{array}{l}\text { General } \\
\text { Salgado }\end{array}$ & T. sordida & 38 & 6 & 15,8 & 244 & 160 & 65,5 & 61 & 13 & 21,3 & 1.118 & 240 & 21,5 \\
\hline Glicério & T. sordida & 22 & 1 & 4,5 & 14 & 6 & 42,8 & 15 & 1 & 6,7 & 70 & 6 & 8,5 \\
\hline Guararapes & T. sordida & 46 & 3 & 6,5 & 62 & 30 & 48,4 & 12 & 4 & 33,3 & 290 & 32 & 11,0 \\
\hline Ilha Solteira & T. sordida & 12 & 1 & 8,3 & 7 & 7 & 100,0 & 1 & 1 & 100,0 & 35 & 12 & 34,3 \\
\hline Itapira* & P. megistus & 16 & 1 & 6,2 & 13 & 4 & 30,8 & 0 & 0 & 0,0 & 65 & 5 & 7,6 \\
\hline Itapura & T. sordida & 8 & 2 & 25,0 & 44 & 9 & 20,4 & 28 & 16 & 57,1 & 216 & 14 & 6,5 \\
\hline Lourdes & T. sordida & 7 & 4 & 57,1 & 152 & 54 & 35,5 & 32 & 7 & 21,8 & 360 & 21 & 5,8 \\
\hline Luziânia & T. sordida & 12 & 1 & 8,3 & 30 & 8 & 26,7 & 7 & 0 & 0,0 & 140 & 8 & 5,7 \\
\hline Piacatu & T. sordida & 11 & 1 & 9,1 & 53 & 18 & 33,9 & 10 & 5 & 50,0 & 252 & 20 & 7,9 \\
\hline Promissão & T. sordida & 37 & 1 & 2,7 & 18 & 3 & 16,7 & 1 & 1 & 100,0 & 45 & 4 & 8,9 \\
\hline $\begin{array}{l}\text { Santo Antônio } \\
\text { do Aracanguá }\end{array}$ & T. sordida & 49 & 1 & 2,1 & 21 & 9 & 42,8 & 2 & 0 & 0,0 & 115 & 9 & 7,8 \\
\hline $\begin{array}{l}\text { Santópolis } \\
\text { do Aguapeí }\end{array}$ & T. sordida & 7 & 1 & 14,3 & 14 & 6 & 42,8 & 11 & 6 & 54,5 & 66 & 6 & 9,0 \\
\hline $\begin{array}{l}\text { São José } \\
\text { de Iracema }\end{array}$ & T. sordida & 9 & 4 & 44,4 & 147 & 26 & 17,7 & 17 & 7 & 41,1 & 225 & 24 & 10,7 \\
\hline Tupi Paulista & T. sordida & 38 & 1 & 2,6 & 28 & 25 & 89,3 & 6 & 1 & 16,7 & 260 & 23 & 8,8 \\
\hline Turiúba & T. sordida & 18 & 1 & 5,5 & 26 & 12 & 46,1 & 6 & 3 & 50,0 & 120 & 11 & 9,2 \\
\hline $\begin{array}{l}\text { Total } \\
\text { (21 municípios) }\end{array}$ & & 481 & 43 & 8,9 & 1.418 & 689 & 48,6 & 400 & 145 & 36,2 & 5.862 & 761 & 12,9 \\
\hline
\end{tabular}

*Município pertencente à Região Administrativa de Saúde da SUCEN de Campinas. 
Apesar de não ter sido realizada integralmente em todas as localidades, a atividade de revisão de controle químico (revisita) obteve percentuais de positividade de $16,9 \%$ para rotina e $24,8 \%$ para notificação.

As casas eram habitadas em $87,4 \%$ das vezes. O tipo de construção predominante foi o tijolo rebocado, tendo sido encontrados ainda $17,7 \%$ das casas em barro ou madeira. Proximidades à mata de cerrado e presença de galinheiros foram observados em $64,3 \%$ e $97,6 \%$ das UDs, respectivamente (Figura 2).

Em 100,0\% das localidades as UDs não apresentaram cuidados de limpeza pelo morador (Figura 3).

\section{Discussão}

O PCDCh no Estado de São Paulo, sob a responsabilidade da SUCEN, está direcionado à vigilância das espécies secundárias. A participação da população para notificar insetos suspeitos de serem triatomíneos tem sido a tônica do PCDCh (Silva et al., 1999). Uma pequena parcela de localidades é visitada regularmente pelas equipes de campo da Sucen, em atividade de rotina. Pudemos observar que, no período de 10 anos, 8,9\% das localidades existentes nos municípios com localidades com alta infestação e $0,3 \%$ do total de localidades do Estado estiveram classificadas em Prioridade 1 (alta infestação). Houve, neste período, uma concentração destas localidades na região de Araçatuba (Figura 1). O serviço de controle da SUCEN nesta região não tem como prioridade o PCDCh, portanto as atividades rotineiras de pesquisa e atendimento às notificações têm sido colocadas em segundo plano. Recorrentes epidemias de dengue na região e agora, mais recentemente, de leishmaniose visceral americana podem ser responsabilizadas por esta situação.

A espécie freqüentemente encontrada nas pesquisas tem sido o T. sordida com predomínio no peridomicílio (infestação peridomiciliar). O hábito alimentar associa-se a sangue de aves, dado seu encontro importante em galinheiros, fato também observado por Silveira \& Vinhaes (1998) em avaliação realizada no Brasil. Isto faz do peridomicílio uma área excelente para formação de colônias de T. sordida, especialmente quando a fonte de alimento é farta, como verificado por Diotaiuti et al. (1994). Lembremos que o peridomicílio normalmente é amplo, com refúgios naturais que servem como abrigo para os triatomíneos e nem sempre ao alcance dos inseticidas e da ação do homem (Diotaiuti, 1991; Diotaiuti et al., 1994; Wander-
Figura 2

Galinheiro. Anexo comum nas localidades

com infestação persistente.

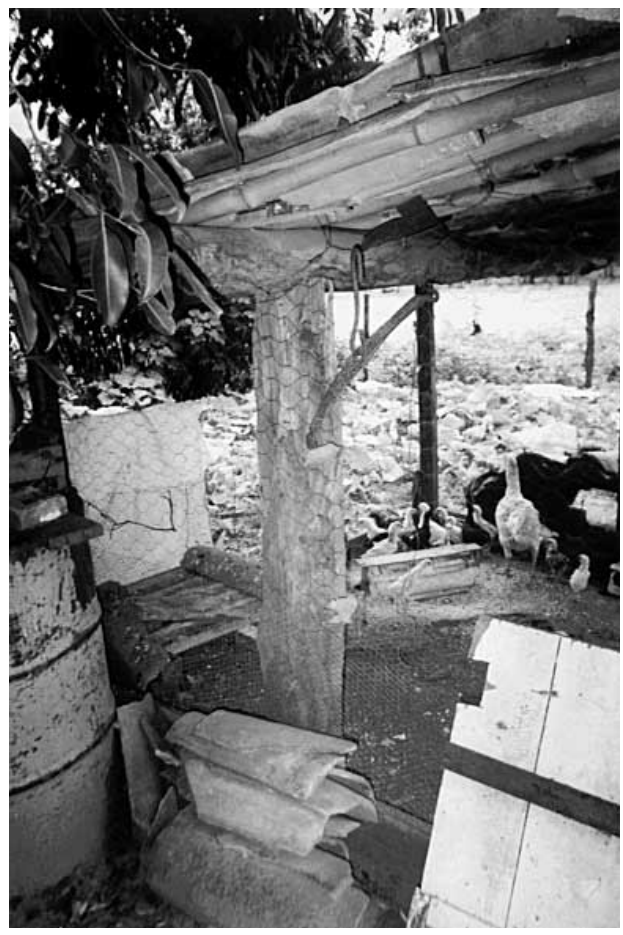

Figura 3

Peridomicílios de área rural. Falta de cuidados pelo morador.

Características observadas em todas as localidades com alta infestação.

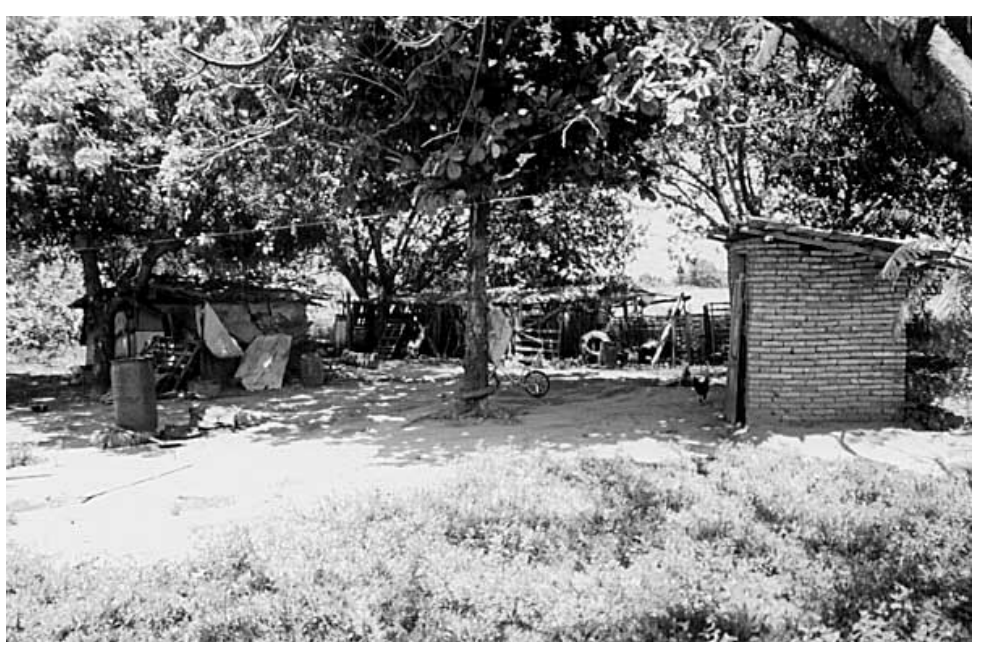


ley, 1994). Neste mesmo ambiente (peridomicílio), a quantidade de alimento disponível é elevada, além do grande trânsito de animais silvestres, que podem estar infectados pelo Trypanosoma cruzi.

O peridomicílio é freqüentemente modificado nestas áreas do estudo, com a destruição de alguns anexos e construção de outros com material vindo diretamente do ambiente silvestre, portanto sem qualquer tratamento. Este fato é relevante, pois o fato de o ambiente estar sempre infestado talvez não possa ser atribuído à falha na ação do inseticida. $\mathrm{Na}$ atividade de revisão de controle químico, 16,9\% das casas pesquisadas em atividade de rotina positivaram novamente e, naquelas pesquisadas em atividade de notificação, este percentual foi de 24,8. De acordo com Dias (1987), o peridomicílio se constitui no mais importante desafio para os programas de controle.

O controle químico nas UDs com encontro de triatomíneo não tem sido realizado concomitantemente à pesquisa triatomínica nesta região, levando cerca de 15 dias para início. Oliveira-Filho et al. (2000), em trabalho realizado com inseticida de ação residual para o controle de T. brasiliensis e T. pseudomaculata no Nordeste brasileiro, alertam para a necessidade de avaliar melhor a aplicação de inseticidas em períodos pequenos, para diminuição da densidade triatomínica no peridomicílio, pois lembram que, muitas vezes, funcionam como uma barreira à entrada de triatomíneos no interior das casas.

A presença de colônias de T. sordida no peridomicílio pode sinalizar para uma iminente colonização desta espécie em futuro próximo, caso as medidas de controle sejam descontinuadas (Dias, 1998; Diotaiuti et al., 1994). Certamente a falta de cuidado da UD pelo morador (Figura 3) e a pouca sensibilização da população em notificar insetos suspeitos de serem triatomíneos são fatores que não se devem descartar. A participação popular no controle dos triatomíneos é um mecanismo eficaz, pois seleciona casas a pesquisar, e com vantagem de acarretar menos custos para o Estado (Silva et al., 1999; Wanderley, 1994). Na área trabalhada, a notificação mostrou-se mais eficaz do que a atividade de rotina (Tabela 1 ). Na atividade de rotina, os triatomíneos são coletados, em sua maioria, no peridomicílio, enquanto que, na atividade de notificação, estes insetos são encaminhados pela população quando da invasão da moradia (intradomicílio) (Silva et al., 1999). O Município de Itapira, região de Campinas, cuja espécie mais freqüentemente coletada é o P. megistus, esteve em todos os biê- nios da década de 1990 com uma localidade classificada em alta infestação (Tabela 1). Nesta localidade, as atividades de pesquisa triatomínica rotineira foram descontinuadas ao longo dos anos.

Em 48,6\% das UDs, a captura de triatomíneos se deu em um único momento da década. Uma relação direta entre o número de triatomíneos coletados e o número de casas positivas revelou a presença, de 34 exemplares por domicílio. Casas construídas de barro ou madeira, $17,7 \%$ do total de casas existentes, associadas ao mau cuidado pelo morador, são ambientes propícios para os triatomíneos.

Quando analisados os diferentes biênios que compuseram a década de 1990, o número de localidades com alta infestação aumenta significativamente e a área de concentração destas passa para a região de São José do Rio Preto, noroeste do Estado de São Paulo. Assim, os resultados do presente estudo podem ser extrapolados a outras áreas rurais do Estado de São Paulo. Podem-se utilizar, para isto, imagens por sensoriamento remoto, permitindo a verificação de características assemelhadas à área original em área muito mais extensa, buscando-se réplicas da microrregião inicialmente caracterizada. De acordo com Hugh-Jones (1989), a combinação dos dados extraídos in loco com os gerados por sensoriamento remoto permitirá visualizar a distribuição de locais da presença de triatomíneos vetores, por exemplo.

Ressaltamos que este mecanismo pode auxiliar nas atividades de controle, demarcandose áreas a serem previamente pesquisadas, porém não se pode perder de vista que o controle integrado será sustentável quando houver uma participação mais efetiva da população na vigilância do peridomicílio. A vigilância entomológica deve ser permanente, exercida pela população, com o máximo aproveitamento dos recursos locais e envolvendo a rede de serviços de saúde, como forma de garantir a manutenção do controle alcançado (Silveira \& Vinhaes, 1998). Outra característica relevante está centrada na realização, durante a pesquisa dos vetores, de um manejo do ambiente de forma adequada, evitando-se que colônias de triatomíneos sejam repassadas de um local para outro, o que permitiria sua persistência dentro do próprio ambiente domiciliar (Rodrigues \& Silva, 2001; Silva \& Rodrigues, 2001).

Uma pesquisa cuidadosa e muito bem realizada, associada ao controle químico e à participação constante da população na notificação de insetos suspeitos serão os mecanismos que garantirão maior sustentabilidade para o PCDCh no Estado de São Paulo. 


\section{Referências}

DIAS, J. C. P., 1987. Control of Chagas disease in Brazil. Parasitology Today, 3:336-341.

DIAS, J. C. P., 1998. Problemas e possibilidades de participação comunitária no controle das grandes endemias no Brasil. Cadernos de Saúde Pública, 14(Sup. 2):19-37.

DIOTAIUTI, L., 1991. Importância e Perspectivas de Controle do Triatoma sordida em Minas Gerais, Belo Horizonte. Tese de Doutorado, Belo Horizonte: Universidade Federal de Minas Gerais.

DIOTAIUTI, L.; PAULA, O. R.; FALCÃO, P. L. \& DIAS, J. C. P., 1994. Evaluation of the Chagas disease vector control program in Minas Gerais, Brazil, with special reference to Triatoma sordida. Bulletin of the Pan American Health Organization, 28:211219.

HUGH-JONES, M. E., 1989. Aplications of remote sensing to the identification of the habitats of parasites and disease vectors. Parasitology Today, 5:244-251.

OLIVEIRA FILHO, A. M.; MELO, M. T. V.; SANTOS, C. E.; FARIA FILHO, O. F.; CARNEIRO, F. C. F.; OLIVEIRA-LIMA, J. W.; VIEIRA, J. B. F.; GADELHA, F. V. \& ISHIHATA, J., 2000. Tratamentos focais e totais com inseticidas de ação residual para o controle de Triatoma brasiliensis e Triatoma pseudomaculata no Nordeste brasileiro. Cadernos de Saúde Pública, 16(Sup. 2):105-111.

RODRIGUES, V. L. C. C. \& SILVA, R. A., 2001. Observações sobre o comportamento dos triatomíneos em situações de manejo do ambiente. Revista da Sociedade Brasileira de Medicina Tropical, 34 (Sup. 3):50-51.

SES-SP (Secretaria de Estado da Saúde de São Paulo), 1989. Relatório Final do Grupo de Trabalho do Programa de Controle da Doença de Chagas no Estado de São Paulo. São Paulo: Superintendência de Controle de Endemias, Secretaria de Estado da Saúde, Governo do Estado de São Paulo.
SILVA, R. A.; BONIFÁCIO, P. R. \& WANDERLEY, D. M. V., 1999. Doença de Chagas no Estado de São Paulo: Comparação entre pesquisa ativa de triatomíneos em domicílios e notificação de sua presença pela população em área de vigilância entomológica. Revista da Sociedade Brasileira de Medicina Tropical, 32:653-659.

SILVA, R. A. \& RODRIGUES, V. L. C. C., 2001. Observações sobre o comportamento da fonte alimentar em situações de manejo do ambiente. Revista da Sociedade Brasileira de Medicina Tropical, 34(Sup. 3):51.

SILVEIRA, A. C. \& VINHAES, M., 1998. Doença de Chagas: Aspectos epidemiológicos e de controle. Revista da Sociedade Brasileira de Medicina Tropical, 31(Sup. 2):15-60.

SOUZA, A. G.; WANDERLEY, D. M. V.; BURALLI, G. M. \& ANDRADE, J. C. R., 1984. Consolidation of the control of Chagas disease in State of São Paulo. Memórias do Instituto Oswaldo Cruz, 79:125-132.

WANDERLEY, D. M. V., 1993. Controle do Triatoma infestans no Estado de São Paulo. Revista da Sociedade Brasileira de Medicina Tropical, 26(Sup. 3):17-25.

WANDERLEY, D. M. V., 1994. Perspectivas de Controle da Doença de Chagas no Estado de São Paulo. Tese de Doutorado, São Paulo: Faculdade de Saúde Pública, Universidade de São Paulo.

Recebido em 12 de julho de 2002

Versão final reapresentada em 25 de outubro de 2002 Aprovado em 5 de fevereiro de 2003 